\title{
No association between dopamine D3 receptor gene Ser9Gly polymorphism (rs6280) and risk of schizophrenia: an updated meta-analysis
}

This article was published in the following Dove Press journal:

Neuropsychiatric Disease and Treatment

Number of times this article has been viewed

\section{Xing-ling Qi \\ Jin-feng Xuan \\ Jia-xin Xing \\ Bao-jie Wang \\ Jun Yao}

School of Forensic Medicine,

China Medical University, Shenyang,

People's Republic of China
Correspondence: Jun Yao

School of Forensic Medicine, China Medical University, Number 77 Puhe

Road, Shenbei New District, Shenyang

I I0I 22, People's Republic of China

Tel +862431939433

Email yaojun198717@163.com
Objective: Ser9Gly (rs6280) is a functional single-nucleotide polymorphism (SNP) in the dopamine receptor D3 (DRD3) gene that may be associated with schizophrenia. We performed a meta-analysis to determine whether Ser9Gly influences the risk of schizophrenia and examined the relationship between the Ser9Gly SNP and the etiology of schizophrenia.

Methods: Case-control studies were retrieved from literature databases in accordance with established inclusion criteria. Odds ratios (ORs) and 95\% confidence intervals (CIs) were calculated to evaluate the strength of the association between Ser9Gly and schizophrenia. Subgroup analysis and sensitivity analysis were also performed.

Results: Seventy-three studies comprising 10,634 patients with schizophrenia (cases) and 11,258 controls were included in this meta-analysis. Summary results indicated no association between Ser9Gly and risk of schizophrenia. In the dominant genetic model, the pooled OR using a random effects model was 0.950 (95\% CI, 0.847-1.064; $P=0.374)$.

Conclusion: Results of this meta-analysis suggest that the Ser9Gly SNP is not associated with schizophrenia. These data provide possible avenues for future case-control studies related to schizophrenia.

Keywords: dopamine receptor D3, schizophrenia, meta-analysis, gene polymorphism

\section{Introduction}

Schizophrenia is a common mental disorder caused by synergic effects of multiple genetic and environmental factors. ${ }^{1}$ Heritability of up to $80 \%$ has been reported for schizophrenia $;{ }^{4}$ however, the precise etiology of this disease remains inconclusive. ${ }^{2,3}$ Results of several genome-wide linkage and association studies have indicated genes and chromosomal regions associated with susceptibility to schizophrenia. ${ }^{5,6}$ Several investigators have suggested that dysregulated dopaminergic neurotransmission has a role in the pathogenesis of schizophrenia..$^{7-10}$ Dopamine functions as a neurotransmitter by binding to dopamine receptors on the postsynaptic membrane and autoreceptors on the presynaptic membrane.

Dopamine receptor D3 (DRD3) is a candidate gene for evaluating an association between dopaminergic neurotransmission and schizophrenia risk. DRD3 is located on chromosome 3 in the q13.3 band and has 52\% global homology with the D2 receptor band. $D R D 3$ is primarily expressed in the limbic areas of the human brain ${ }^{11}$ and contributes emotional, cognitive, and endocrine functions. ${ }^{12}$ A single-nucleotide polymorphism (SNP) in the first exon of $D R D 3$ corresponds to a serine-to-glycine substitution at position 9 in the extracellular N-terminal domain of the polypeptide 
(ie, Ser9Gly [rs6280]). Ser9Gly is a functional SNP that yields a protein with altered dopamine-binding affinity. ${ }^{13}$ The substitution of serine with glycine is thought to yield D3 autoreceptors with a higher affinity for dopamine and more robust intracellular signaling. ${ }^{14}$ Other authors have associated Ser9Gly with acute pain in sickle cell disease, bipolar disorder, Parkinson's disease, and suicidal behaviors. ${ }^{15-18}$

In recent years, numerous molecular epidemiological studies have addressed the association between Ser9Gly and schizophrenia risk. However, some investigators determined that Ser9Gly was associated with the disease, ${ }^{19,20}$ whereas others found no association. ${ }^{21-23}$ These inconclusive and discordant findings have been attributed to small sample size, inclusion of various genetic backgrounds, and potential confounding bias. ${ }^{24}$

Meta-analysis has been applied widely as a statistical method in medical studies, particularly for topics that are studied extensively yet yield controversial results. ${ }^{25}$ Utsunomiya et al conducted a meta-analysis in 2008 to evaluate the association between Ser9Gly and schizophrenia. ${ }^{26}$ Their pooled results of 9 case-control studies indicated that Ser9Gly was unlikely to confer susceptibility to schizophrenia in the Japanese population. ${ }^{26}$ In a second meta-analysis conducted in 2008, results involving 51 case-control studies indicated no association of Ser9Gly with schizophrenia. ${ }^{21}$ In the years since these meta-analyses were completed, additional molecular epidemiological studies have addressed the roles of Ser9Gly in the occurrence of schizophrenia in various populations. Herein, we describe an updated metaanalysis of studies involving associations between DRD3 polymorphisms and schizophrenia.

\section{Methods}

\section{Identification of relevant studies}

To identify studies eligible for inclusion in this meta-analysis, 3 online electronic English databases (PubMed, Embase, and Web of Science) and 1 online Chinese database (CNKI) were searched. The most recent search was conducted in July 2017. The following key words were used for study identification: DRD3, dopamine receptor 3, dopamine D3 receptor, dopamine receptor D3, schizophrenia, polymorphism, and Ser9Gly. Reference lists of the accessed articles and of potentially relevant review articles were screened to identify additional studies.

The following inclusion criteria were applied: 1) casecontrol design; 2) inclusion of patients with schizophrenia; and 3) statement of allele or genotype frequencies. For studies in which the same or overlapping data were reported by the same authors, the most recent article was selected. Excluded from the meta-analysis were studies 1) without a control population, 2) that duplicated an earlier publication, and 3 ) that lacked data regarding genotype frequency. Study authors were queried via e-mail for additional study details, such as allele or genotype frequencies or sample characteristics, when these data were not provided in the article.

\section{Data extraction}

Two reviewers independently extracted information from all eligible publications. Disagreements were resolved by discussion until the 2 reviewers reached consensus. The following details of each article were recorded: first author's last name, publication year, sample size, region, and number of genotypes for cases and controls. To detect potentially moderating influences on the effects findings reported in the case-control studies, we also included the following variables: 1) ethnicity of the sample population; 2) source of controls; 3) mean age of the control group; 4) diagnostic criteria; and 5) gender index.

\section{Statistical analysis}

Stata version 10.0 (Stata Corp., College Station, TX, USA) was applied for statistical analysis. Hardy-Weinberg equilibrium (HWE) was determined for the genotype distribution of controls, and the chi-square goodness-of-fit test was performed to ascertain deviations from HWE. The Thakkinstian method was applied for pooled frequency analysis, as described previously. ${ }^{27}$ All statistical tests were 2-tailed, and significance was defined as $P<0.05$.

Odds ratios (ORs) with accompanying 95\% confidence intervals (CIs) were calculated to assess the strength of the association of Ser9Gly and schizophrenia. Pooled effect sizes among the included articles were examined with a random effects model, which accounts for heterogeneity among the studies and yields the likely effect size across populations. We did not apply a fixed effects model because we wanted to avoid the assumption that patients were being sampled from a single population. In the fixed effects model, the effect size could be biased by heterogeneity among studies. ${ }^{28}$

Three genetic models were applied to determine overall pooled ORs: the allele contrast model, the dominant model, and the recessive model. As previously described, $\mathrm{OR}_{1}$ (AA vs aa), OR (Aa vs aa), and $\mathrm{OR}_{3}$ (AA vs Aa) were compared, with $\mathrm{A}$ defined as the risk allele. ${ }^{25}$ The most suitable genetic model was ascertained from these pairwise differences. Specifically, for $\mathrm{OR}_{1}=\mathrm{OR}_{3} \neq 1$ and $\mathrm{OR}_{2}=1$, the recessive model was selected $(\mathrm{OR}=1$ means $P>0.05$; 
$\mathrm{OR} \neq 1$ means $P<0.05)$. For $\mathrm{OR}_{1}=\mathrm{OR}_{2} \neq 1$ and $\mathrm{OR}_{3}=1$, the dominant model was considered. For $\mathrm{OR}_{2}=1 / \mathrm{OR}_{3} \neq 1$ and $\mathrm{OR}_{1}=1$, the complete-overdominant model was presumed. Lastly, for $\mathrm{OR}_{1}>\mathrm{OR}_{2}>1$ and $\mathrm{OR}_{1}>\mathrm{OR}_{3}>1$ ( or $\mathrm{OR}_{1}<\mathrm{OR}_{2}<1$ and $\mathrm{OR}_{1}<\mathrm{OR}_{3}<1$ ), the data were evaluated in the context of the codominant model. ${ }^{29}$

The degree of heterogeneity between studies was determined by means of the $Q$ statistic. ${ }^{30,31}$ Specifically, $P>0.05$ by the $Q$ test indicated the absence of heterogeneity, and $P<0.05$ indicated heterogeneity. $I^{2}$ was defined as the proportion of observed variance in effect sizes attributable to true differences among studies. Conventional interpretations of $I^{2}$ include limits for low $(<25 \%)$, moderate (approximately $50 \%$ ), and high $(>75 \%)$ heterogeneity. ${ }^{32}$ Subgroup analysis was carried out by ethnicity (ie, East Asian, Caucasian, and other populations) and by source of controls (ie, hospitalbased and population-based).

Publication bias was evaluated by visual inspection of a funnel plot in which the standard error of $\log (\mathrm{OR})$ of each study was plotted against its $\log (\mathrm{OR})$. An asymmetric plot implied possible publication bias, and the degree of asymmetry was calculated by means of Egger's test. $P<0.05$ indicated significant publication bias. ${ }^{33}$
Sensitivity analysis was performed to assess the potential influence of a single study on the pooled effect size. Specifically, each study was omitted singly from the meta-analysis, and significant alterations to the pooled effect size were ascertained.

\section{Results}

A total of 155 articles were identified by database searches. After removing duplicate or overlapping articles and those that did not fulfill the inclusion criteria, 60 publications were included in the meta-analysis..$^{12,19-23,26,34-85}$ These articles included 73 individual studies that comprised 10,634 patients with schizophrenia (ie, cases) and 11,258 unaffected participants (ie, controls). Patients of diverse races and ethnicities were included (eg, East Asian, Caucasian, Latino, and Indian). The mean age of the controls ranged from 25.0 to 53.0 years. The key characteristics of the studies are summarized in Table 1. Genotype and allele frequencies, and details regarding HWE are presented in Table 2. For Ser9Gly, the total numbers of Ser/Ser, Ser/Gly, and Gly/Gly genotypes were 5,532, 5,117, and 1,900 for cases and 5,173, 5,066, and 1,022 for controls, respectively. Of the 73 studies, 4 studies deviated significantly from HWE.

Table I Baseline characteristics of qualified studies in this meta-analysis

\begin{tabular}{|c|c|c|c|c|c|c|c|c|}
\hline References & Year & Location & Ethnicity & $\begin{array}{l}\text { Controls } \\
\text { source }\end{array}$ & $\begin{array}{l}\text { Mean age } \\
\text { of control } \\
\text { group }\end{array}$ & $\begin{array}{l}\text { Diagnostic } \\
\text { criteria }\end{array}$ & $\begin{array}{l}\text { Gender } \\
\text { index } \\
\text { (case) }\end{array}$ & $\begin{array}{l}\text { Gender } \\
\text { index } \\
\text { (control) }\end{array}$ \\
\hline Crocq et al ${ }^{19}$ & 1992 & France & Caucasian & Hospital-based & 33.9 & DSM-III-R & 0.38 & - \\
\hline Crocq et $\mathrm{al}^{19}$ & 1992 & UK & Caucasian & Population-based & 45.9 & DSM-III-R & 0.58 & 0.74 \\
\hline Yang et $\mathrm{al}^{61}$ & 1993 & China & East Asians & Population-based & 25.05 & RDC & 0.49 & 0.56 \\
\hline Nanko et $\mathrm{al}^{64}$ & 1993 & Japan & East Asians & Population-based & 27.8 & DSM-III-R & 0.82 & 0.91 \\
\hline Jönsson et $\mathrm{al}^{67}$ & 1993 & Sweden & Caucasian & Population-based & 39 & DSM-III-R & 0.46 & 0.61 \\
\hline Nöthen et $\mathrm{al}^{72}$ & 1993 & Germany & Caucasian & Population-based & - & - & - & - \\
\hline Nöthen et $\mathrm{al}^{73}$ & 1993 & Germany & Caucasian & Population-based & 28.2 & DSM-III-R & 0.5 & 0.88 \\
\hline Laurent et al ${ }^{45}$ & 1994 & France & Caucasian & Population-based & 48 & DSM-III-R & 0.38 & 0.72 \\
\hline Saha et $\mathrm{al}^{53}$ & 1994 & Singapore & East Asians & Population-based & 38 & ICD-9 & - & - \\
\hline Mant et $\mathrm{al}^{65}$ & 1994 & UK & Caucasian & Population-based & 46.6 & DSM-III-R & 0.74 & 0.8 \\
\hline Kennedy et $\mathrm{al}^{66}$ & 1995 & North America & Caucasian & Hospital-based & - & DSM-III-R & - & - \\
\hline Kennedy et al ${ }^{66}$ & 1995 & Italy & Caucasian & Hospital-based & - & DSM-III-R & - & - \\
\hline Inada et $\mathrm{al}^{81}$ & 1995 & Japan & East Asians & Population-based & 54 & - & 1.09 & 1 \\
\hline Durany et $\mathrm{al}^{38}$ & 1996 & Spain & Caucasian & Population-based & 53 & ICD-I0 & 1.38 & 1.44 \\
\hline Gaitonde et $\mathrm{al}^{41}$ & 1996 & UK & Caucasian & Hospital-based & 41.7 & ND & 0.83 & 0.93 \\
\hline Ohara et $\mathrm{al}^{50}$ & 1996 & Japan & East Asians & Population-based & 34.4 & DSM-IV & - & 1.37 \\
\hline Rietschel et $\mathrm{al}^{51}$ & 1996 & Germany & Caucasian & Population-based & 30.2 & DSM-III-R & 0.66 & 0.96 \\
\hline Shaikh et $\mathrm{a}^{54}$ & 1996 & UK & Caucasian & Hospital-based & - & DSM-III-R & - & - \\
\hline Tanaka et $\mathrm{al}^{59}$ & 1996 & Japan & East Asians & Population-based & 42.7 & DSM-III-R & 0.92 & 0.41 \\
\hline Nimgaonkar et $\mathrm{al}^{20}$ & 1996 & USA & African-American & Hospital-based & - & DSM-III-R & 1.24 & 1.33 \\
\hline Nimgaonkar et $\mathrm{al}^{20}$ & 1996 & USA & Caucasian & Hospital-based & - & DSM-III-R & 0.67 & I.I \\
\hline Chen et $\mathrm{al}^{22}$ & 1997 & China & East Asians & Hospital-based & 45 & DSM-III-R & 0.86 & 1.13 \\
\hline Ebstein et $\mathrm{al}^{39}$ & 1997 & Italy & Caucasian & Population-based & 36.5 & DSM-III-R & 0.31 & 1.03 \\
\hline Ebstein et $\mathrm{al}^{39}$ & 1997 & Israel & Ashkenazi & Population-based & 32.9 & DSM-III-R & - & 0.94 \\
\hline
\end{tabular}


Table I (Continued)

\begin{tabular}{|c|c|c|c|c|c|c|c|c|}
\hline References & Year & Location & Ethnicity & $\begin{array}{l}\text { Controls } \\
\text { source }\end{array}$ & $\begin{array}{l}\text { Mean age } \\
\text { of control } \\
\text { group }\end{array}$ & $\begin{array}{l}\text { Diagnostic } \\
\text { criteria }\end{array}$ & $\begin{array}{l}\text { Gender } \\
\text { index } \\
\text { (case) }\end{array}$ & $\begin{array}{l}\text { Gender } \\
\text { index } \\
\text { (control) }\end{array}$ \\
\hline Ebstein et al ${ }^{39}$ & 1997 & Israel & Non-Ashkenazi & Population-based & 32.9 & DSM-III-R & - & 0.94 \\
\hline Maziade et $\mathrm{al}^{48}$ & 1997 & Canada & Caucasian & Population-based & - & DSM-III-R & 0.46 & - \\
\hline Hawi et $\mathrm{al}^{42}$ & 1998 & Ireland & Caucasian & Population-based & - & DSM-III-R & 0.47 & 0.79 \\
\hline Krebs et $\mathrm{al}^{92}$ & 1998 & France & Caucasian & Population-based & 35.47 & DSM-III-R & 0.62 & I \\
\hline Spurlock et al ${ }^{56}$ & 1998 & Ireland & Caucasian & Population-based & - & DSM-III-R & - & - \\
\hline \multirow[t]{2}{*}{ Spurlock et al ${ }^{56}$} & 1998 & Northern & Caucasian & Population-based & - & DSM-III-R & - & - \\
\hline & & Sweden & & & & & & \\
\hline Spurlock et $\mathrm{a}^{56}$ & 1998 & Portugal & Caucasian & Population-based & - & DSM-III-R & - & - \\
\hline Spurlock et $\mathrm{al}^{56}$ & 1998 & Wales & Caucasian & Population-based & - & DSM-III-R & - & - \\
\hline Spurlock et al ${ }^{56}$ & 1998 & Austria & Caucasian & Population-based & - & DSM-III-R & - & - \\
\hline Spurlock et al ${ }^{56}$ & 1998 & France & Caucasian & Population-based & - & DSM-III-R & - & - \\
\hline Ishiguro et $\mathrm{al}^{43}$ & 2000 & Japan & East Asians & Population-based & 47.2 & DSM-III-R or ICD-I0 & 0.74 & 1.07 \\
\hline Ishiguro et al ${ }^{43}$ & 2000 & Japan & East Asians & Population-based & 48.5 & DSM-III-R or ICD-II & 0.9 & 0.81 \\
\hline Joober et $\mathrm{al}^{44}$ & 2000 & Canada & Caucasian & Hospital-based & - & DSM-IV & - & - \\
\hline Meszaros et al ${ }^{49}$ & 2000 & Austria & Caucasian & Population-based & - & DSM-III-R & - & - \\
\hline $\begin{array}{l}\text { Sivagnanasundaram } \\
\text { et a }\left.\right|^{55}\end{array}$ & 2000 & UK & Caucasian & Population-based & - & DSM-III-R & - & - \\
\hline Hauser et $\mathrm{al}^{77}$ & 2000 & Poland & Caucasian & Population-based & 28.76 & DSM-IV & - & - \\
\hline Cordeiro et $\mathrm{al}^{37}$ & 2001 & Brazil & Latinos & Population-based & - & ICD-I0 & - & - \\
\hline Løvlie et $\mathrm{a}^{47}$ & 2001 & India & Indians & Population-based & 43 & DSM-IV & - & 0.83 \\
\hline Rybakowski et $\mathrm{al}^{52}$ & 2001 & Poland & Caucasian & Population-based & 27 & DSM-IV, ICD-I0 & 0.61 & 1.13 \\
\hline Anney et $\mathrm{a}^{35}$ & 2002 & UK and Ireland & Caucasian & Population-based & 43 & DSM-IV & 0.28 & 0.28 \\
\hline Ventriglia et a $\left.\right|^{60}$ & 2002 & Italy & Caucasian & Population-based & - & DSM-IV & - & - \\
\hline Morimoto et $\mathrm{a}^{62}$ & 2002 & Japan & East Asians & Population-based & - & ICD-I0 & 1.14 & - \\
\hline Zhao et $\mathrm{a}^{83}$ & 2002 & China & East Asians & Population-based & 55.9 & DSM-III-R & 0.83 & 1.4 \\
\hline Tang et $\mathrm{al}^{84}$ & 2002 & China & East Asians & Population-based & 33 & CCMD-II-R & 0.76 & 1.06 \\
\hline Jönsson et $\mathrm{al}^{71}$ & 2003 & Sweden & Caucasian & Population-based & - & DSM-III-R & - & - \\
\hline $\mid$ wata et $\mathrm{al}^{76}$ & 2003 & Japan & East Asians & Population-based & - & DSM-IV & - & - \\
\hline Baritaki et $\mathrm{al}^{36}$ & 2004 & Greece & Caucasian & Population-based & 45.1 & DSM-IV & 0.7 & 0.63 \\
\hline Jönsson et $\mathrm{a}^{63}$ & 2004 & Germany & Caucasian & Population-based & 30.2 & DSM-IV & 0.85 & 0.25 \\
\hline$A$ et $\mathrm{al}^{82}$ & 2004 & China & East Asians & Population-based & - & - & 0.63 & - \\
\hline Staddon et al ${ }^{57}$ & 2005 & Northern Spain & Basque & Population-based & - & DSM-IV & 0.54 & $\mathrm{I}$ \\
\hline Yang ${ }^{93}$ & 2005 & China & East Asians & Population-based & 35.04 & DSM-IV & 1.12 & 1.09 \\
\hline Liang $^{94}$ & 2005 & China & East Asians & Population-based & 25 & DSM-IV, CCMD-3 & 0.98 & 0.98 \\
\hline Talkowski et a ${ }^{58}$ & 2006 & USA & Caucasian & Population-based & - & DSM-IV & - & - \\
\hline Yi et a ${ }^{85}$ & 2006 & China & East Asians & Population-based & 35 & DSM-IV & 1.12 & 1.13 \\
\hline Ma et $\mathrm{al}^{21}$ & 2008 & China & East Asians & Hospital-based & 35.02 & DSM-IV & 0.62 & 0.81 \\
\hline Lorenzo et al ${ }^{46}$ & 2007 & Spain & Caucasian & Population-based & - & DSM-IV & - & - \\
\hline Chang et $\mathrm{al}^{68}$ & 2007 & China & East Asians & Population-based & - & DSM-IV & - & - \\
\hline Güzey et $\mathrm{al}^{34}$ & 2007 & Italy & Caucasian & Population-based & - & DSM-IV & 0.2 & 0.17 \\
\hline Fathalli et $\mathrm{al}^{40}$ & 2008 & $\begin{array}{l}\text { Canada, Tunisia, } \\
\text { and Hungary }\end{array}$ & Caucasian & Hospital-based & - & $\begin{array}{l}\text { DSM-III-R or } \\
\text { DSM-IV }\end{array}$ & 0.37 & 0.85 \\
\hline Utsunomiya et $\mathrm{a}^{26}$ & 2008 & Japan & East Asians & Population-based & 55 & DSM-IV & 0.92 & 0.92 \\
\hline Krelling et $\mathrm{al}^{78}$ & 2008 & Brazil & Latinos & Population-based & 40.27 & - & - & - \\
\hline Barlas et $\mathrm{al}^{23}$ & 2009 & Turkey & Caucasian & Population-based & 31.7 & DSM-IV & 0.21 & 0.23 \\
\hline Zai et a $\left.\right|^{69}$ & 2010 & Europe & Caucasian & Population-based & - & DSM-IV & 0.57 & 0.42 \\
\hline Sáiz et $\mathrm{al}^{75}$ & 2010 & $\begin{array}{l}\text { Asturia, } \\
\text { Northern Spain }\end{array}$ & Caucasian & Population-based & 40.6 & DSM-IV & 0.66 & 0.95 \\
\hline Nunokawa et a ${ }^{80}$ & 2010 & Japan & East Asians & Population-based & 38.1 & DSM-IV & 0.9 & 0.92 \\
\hline Zhang et $\mathrm{al}^{70}$ & 2011 & China & East Asians & Population-based & 28.13 & DSM-IV & - & - \\
\hline Tee et $\mathrm{al}^{74}$ & 2011 & Malaysia & East Asians & Population-based & 38.4 & - & 0.91 & 0.83 \\
\hline Zheng et $\mathrm{al}^{79}$ & 2012 & China & East Asians & Population-based & 33.1 & DSM-IV & 0.69 & 0.72 \\
\hline Yang et al ${ }^{12}$ & 2016 & China & East Asians & Population-based & 42 & DSM-IV & - & - \\
\hline
\end{tabular}

Notes: Gender index = (female/male). En dashes indicate data not available.

Abbreviations: DSM, Diagnostic and Statistical Manual of Mental Disorders; RDC, Research Diagnostic Criteria; ICD, International Classification of Diseases; ND, not determined; CCMD, Chinese Classification of Mental Disorders. 
Table 2 Distribution of genotype and allele frequencies of the DRD3 Ser9Gly polymorphism

\begin{tabular}{|c|c|c|c|c|c|c|c|c|c|c|c|}
\hline \multirow[t]{3}{*}{ References } & \multicolumn{6}{|c|}{ Genotype distribution } & \multirow[t]{3}{*}{$P_{\text {HWE }}$} & \multicolumn{4}{|c|}{ Allele frequency } \\
\hline & \multicolumn{3}{|l|}{ Cases, $\mathbf{n}$} & \multicolumn{3}{|c|}{ Controls, $n$} & & \multicolumn{2}{|c|}{ Cases, \% } & \multicolumn{2}{|c|}{ Controls, \% } \\
\hline & Ser/Ser & Ser/Gly & Gly/Gly & Ser/Ser & Ser/Gly & Gly/Gly & & Ser & Gly & Ser & Gly \\
\hline Crocq et al ${ }^{19}$ & 37 & 26 & 10 & 134 & 128 & 24 & 0.3930 & 68 & 32 & 69 & 31 \\
\hline Crocq et al ${ }^{19}$ & 37 & 18 & 13 & 170 & 153 & 41 & 0.4616 & 67 & 33 & 68 & 32 \\
\hline Yang et $a^{61}$ & 54 & 45 & 8 & 56 & 95 & 24 & 0.1630 & 65 & 35 & 59 & 41 \\
\hline Nanko et $a^{64}$ & 48 & 35 & 8 & 50 & 40 & 10 & 0.6300 & 72 & 28 & 70 & 30 \\
\hline Jönsson et $\mathrm{al}^{67}$ & 34 & 36 & 6 & 63 & 83 & 37 & 0.3154 & 60 & 40 & 55 & 45 \\
\hline Nöthen et $\mathrm{al}^{72}$ & 31 & 22 & 7 & 26 & 41 & 4 & 0.0193 & 68 & 32 & 65 & 35 \\
\hline Nöthen et $\mathrm{al}^{73}$ & 20 & 26 & 14 & 25 & 34 & 9 & 0.6289 & 68 & 32 & 62 & 38 \\
\hline Laurent et $\mathrm{al}^{45}$ & 35 & 33 & 8 & 43 & 47 & 10 & 0.5832 & 70 & 30 & 67 & 33 \\
\hline Saha et $\mathrm{a}^{53}$ & 62 & 66 & 9 & 34 & 25 & 4 & $0.834 I$ & 66 & 34 & 74 & 26 \\
\hline Mant et $\mathrm{a}^{65}$ & 33 & 23 & 10 & 62 & 41 & 6 & 0.8178 & 77 & 23 & 76 & 24 \\
\hline Kennedy et $\mathrm{al}^{66}$ & 37 & 62 & 18 & 12 & 14 & I & 0.2059 & 61 & 39 & 70 & 30 \\
\hline Kennedy et al ${ }^{66}$ & 42 & 43 & 12 & 73 & 84 & 15 & 0.1807 & 63 & 37 & 67 & 33 \\
\hline Inada et $\mathrm{al}^{81}$ & 66 & 40 & 7 & 34 & 33 & 10 & 0.6569 & 67 & 33 & 66 & 34 \\
\hline Durany et $\mathrm{al}^{38}$ & 53 & 43 & 11 & 92 & 119 & 24 & 0.1064 & 64 & 36 & 64 & 36 \\
\hline Gaitonde et $\mathrm{a}^{41}$ & 34 & 45 & 5 & 56 & 51 & 15 & 0.5255 & 75 & 25 & 67 & 33 \\
\hline Ohara et $\mathrm{al}^{50}$ & 1 & 152 & 0 & 59 & 58 & 15 & 0.8961 & 77 & 23 & 67 & 33 \\
\hline Rietschel et $\mathrm{al}^{51}$ & 61 & 71 & 14 & 42 & 43 & 4 & 0.0865 & 65 & 35 & 71 & 29 \\
\hline Shaikh et $\mathrm{al}^{54}$ & 33 & 56 & 20 & 20 & 27 & 5 & 0.3386 & 65 & 35 & 64 & 36 \\
\hline Tanaka et $\mathrm{al}^{59}$ & 54 & 38 & 8 & 37 & 40 & 9 & 0.707 & 69 & 31 & 66 & 34 \\
\hline Nimgaonkar et $\mathrm{al}^{20}$ & 30 & 22 & 13 & 51 & 66 & 15 & 0.3559 & 67 & 33 & 64 & 36 \\
\hline Nimgaonkar et $\mathrm{al}^{20}$ & 33 & 26 & 6 & 5 & 13 & 4 & 0.3874 & 54 & 46 & 52 & 48 \\
\hline Chen et $\mathrm{a}^{22}$ & 89 & 77 & 12 & 38 & 35 & 6 & 0.5939 & 78 & 22 & 70 & 30 \\
\hline Ebstein et $\mathrm{al}^{39}$ & 37 & 31 & 12 & 49 & 58 & 13 & 0.4951 & 66 & 34 & 65 & 35 \\
\hline Ebstein et $\mathrm{a}^{39}$ & 24 & 15 & 2 & 3 & 118 & 0 & - & 75 & 25 & 76 & 24 \\
\hline Ebstein et $\mathrm{al}^{39}$ & 20 & 16 & 10 & 49 & 42 & 9 & 1 & 66 & 34 & 70 & 30 \\
\hline Maziade et $\mathrm{al}^{48}$ & $4 I$ & 27 & 2 & 54 & 34 & 6 & 0.8354 & 69 & 31 & 76 & 24 \\
\hline Hawi et $\mathrm{al}^{42}$ & 83 & 87 & 28 & 59 & 57 & 9 & 0.3379 & 70 & 30 & 69 & 31 \\
\hline Krebs et $\mathrm{a}^{92}$ & 36 & 42 & 11 & 57 & 69 & 7 & 0.0163 & 66 & 34 & 56 & 44 \\
\hline Spurlock et al $\left.\right|^{56}$ & 15 & 16 & 5 & 25 & 23 & 8 & 0.4763 & 36 & 64 & 83 & 17 \\
\hline Spurlock et al ${ }^{56}$ & 25 & 29 & 13 & 28 & 49 & 8 & 0.042 & 64 & 36 & 62 & 38 \\
\hline Spurlock et $\mathrm{al}^{56}$ & 28 & 40 & 8 & 27 & 34 & 10 & 0.8928 & 59 & 41 & 62 & 38 \\
\hline Spurlock et al $\left.\right|^{56}$ & 14 & 15 & 2 & 6 & 22 & 5 & 0.0546 & 63 & 37 & 51 & 49 \\
\hline Spurlock et al $\left.\right|^{56}$ & 38 & 21 & 12 & 13 & 16 & 2 & 0.3137 & 69 & 31 & 68 & 32 \\
\hline Spurlock et al $\left.\right|^{56}$ & 17 & 11 & 2 & 23 & 28 & 6 & 0.554 & 68 & 32 & 65 & 35 \\
\hline Ishiguro et al ${ }^{43}$ & 84 & 61 & 8 & 10 & 17 & 4 & 0.4375 & 75 & 25 & 60 & 40 \\
\hline Ishiguro et $\mathrm{al}^{43}$ & 61 & 31 & 7 & 67 & 77 & 12 & 0.1118 & 72 & 28 & 69 & 31 \\
\hline Joober et al ${ }^{44}$ & 44 & 50 & 12 & 119 & 127 & 26 & 0.3435 & 75 & 25 & 67 & 33 \\
\hline Meszaros et $\mathrm{al}^{49}$ & 45 & 35 & 15 & 52 & 43 & 5 & 0.2991 & 73 & 27 & 74 & 26 \\
\hline Sivagnanasundaram et $\mathrm{a}^{55}$ & 29 & 40 & 4 & 59 & 67 & 12 & 0.2476 & 60 & 40 & 67 & 33 \\
\hline Hauser et $\mathrm{al}^{77}$ & 62 & 58 & 9 & 50 & 40 & 8 & I & 71 & 29 & 71 & 29 \\
\hline Cordeiro et $\mathrm{a}^{37}$ & 56 & 57 & 28 & 19 & 25 & 4 & 0.2847 & 70 & 30 & 66 & 34 \\
\hline Løvlie et $\mathrm{al}^{47}$ & 16 & 29 & 11 & 291 & 242 & 51 & 0.9456 & 70 & 30 & 71 & 29 \\
\hline Rybakowski et a $\left.\right|^{52}$ & 54 & 55 & 10 & 48 & 35 & 7 & 0.8604 & 72 & 28 & 73 & 27 \\
\hline Anney et $\mathrm{a}^{35}$ & 152 & 178 & 30 & 38 & 46 & 13 & 0.8753 & 67 & 33 & 63 & 37 \\
\hline Ventriglia et $\mathrm{a}^{60}$ & 43 & 51 & 20 & 88 & 81 & 19 & 0.9546 & 59 & 41 & 69 & 31 \\
\hline Morimoto et a $\mathrm{l}^{62}$ & 23 & 21 & 4 & 34 & 26 & 4 & 0.7411 & 65 & 35 & 73 & 27 \\
\hline Zhao et al ${ }^{83}$ & 109 & 109 & 18 & 27 & 22 & 4 & 0.8681 & 68 & 32 & 72 & 28 \\
\hline Tang et $\mathrm{al}^{84}$ & 273 & 210 & 45 & 138 & 119 & 28 & 0.7518 & 67 & 33 & 69 & 31 \\
\hline Jönsson et $\mathrm{al}^{71}$ & 72 & 70 & 14 & 30 & 30 & 3 & 0.1859 & 63 & 37 & 71 & 29 \\
\hline Iwata et $\mathrm{a}^{76}$ & 73 & 64 & 9 & 27 & 30 & 8 & $0.940 \mathrm{I}$ & 71 & 29 & 65 & 35 \\
\hline Baritaki et $\mathrm{al}^{36}$ & 51 & 46 & 17 & 70 & 66 & 27 & 0.098 & 66 & 34 & 63 & 37 \\
\hline Jönsson et al ${ }^{63}$ & 326 & 255 & 68 & 50 & 37 & 7 & 0.9657 & 70 & 30 & 73 & 23 \\
\hline A et $\mathrm{al}^{82}$ & 43 & 29 & 8 & 27 & 21 & 7 & 0.3735 & 71 & 29 & 68 & 32 \\
\hline Staddon et $\mathrm{al}^{57}$ & 59 & 40 & 10 & 278 & 267 & 51 & 0.2413 & 72 & 28 & 69 & 31 \\
\hline
\end{tabular}


Table 2 (Continued)

\begin{tabular}{|c|c|c|c|c|c|c|c|c|c|c|c|}
\hline \multirow[t]{3}{*}{ References } & \multicolumn{6}{|c|}{ Genotype distribution } & \multirow{3}{*}{$\boldsymbol{P}_{\text {HWE }}$} & \multicolumn{4}{|c|}{ Allele frequency } \\
\hline & \multicolumn{3}{|l|}{ Cases, $\mathbf{n}$} & \multicolumn{3}{|c|}{ Controls, n } & & \multicolumn{2}{|c|}{ Cases, \% } & \multicolumn{2}{|c|}{ Controls, $\%$} \\
\hline & Ser/Ser & Ser/Gly & Gly/Gly & Ser/Ser & Ser/Gly & Gly/Gly & & Ser & Gly & Ser & Gly \\
\hline Yang ${ }^{93}$ & 35 & 28 & 7 & 377 & 341 & 50 & 0.019 & 70 & 30 & 71 & 29 \\
\hline Liang $^{94}$ & 65 & 30 & 6 & 213 & 193 & 36 & 0.3993 & 69 & 31 & 70 & 30 \\
\hline Talkowski et al ${ }^{58}$ & 173 & 136 & 12 & 28 & 27 & 5 & 0.6699 & 70 & 30 & 69 & 31 \\
\hline Yi et al $^{85}$ & 35 & 28 & 7 & 14 & 30 & 16 & 0.9931 & 55 & 45 & 48 & 52 \\
\hline Ma et $\mathrm{al}^{21}$ & 145 & 157 & 7 & 47 & 34 & 9 & 0.4449 & 72 & 28 & 71 & 29 \\
\hline Lorenzo et al ${ }^{46}$ & 78 & 82 & 18 & 66 & 78 & 13 & 0.1281 & 67 & 34 & 67 & 33 \\
\hline Chang et $\mathrm{al}^{68}$ & 120 & 105 & 31 & 115 & 75 & 8 & 0.3241 & 69 & 32 & 77 & 23 \\
\hline Güzey et $\mathrm{al}^{34}$ & 30 & 29 & 4 & 164 & 188 & 43 & 0.3158 & 62 & 38 & 65 & 35 \\
\hline Fathalli et al ${ }^{40}$ & 158 & 199 & 51 & 39 & 45 & 16 & 0.619 & 71 & 29 & 62 & 39 \\
\hline Utsunomiya et $\mathrm{a}^{26}$ & 120 & 97 & 29 & 26 & 15 & 7 & 0.0729 & 72 & 28 & 70 & 30 \\
\hline Krelling et $\mathrm{al}^{78}$ & 22 & 56 & 25 & 65 & 39 & 7 & $0.725 \mathrm{I}$ & 71 & 30 & 76 & 24 \\
\hline Barlas et $\mathrm{al}^{23}$ & 47 & 37 & 8 & 15 & 26 & 20 & 0.2682 & 49 & 52 & 46 & 54 \\
\hline Zai et $\mathrm{al}^{69}$ & 66 & 82 & 15 & 177 & 162 & 24 & 0.1038 & 69 & 31 & 71 & 29 \\
\hline Sáiz et $\mathrm{al}^{75}$ & 103 & 123 & 39 & 306 & 243 & 46 & 0.815 & 7I & 29 & 72 & 28 \\
\hline Nunokawa et $\mathrm{al}^{80}$ & 301 & 239 & 54 & 28 & 19 & I & 0.2734 & 76 & 24 & 78 & 22 \\
\hline Zhang et $\mathrm{al}^{70}$ & 345 & 274 & 66 & 52 & 42 & $\mathrm{II}$ & 0.5655 & 79 & 21 & 70 & 31 \\
\hline Tee et $\mathrm{al}^{74}$ & 120 & 107 & 34 & 153 & 145 & 17 & 0.0195 & 69 & 31 & 72 & 28 \\
\hline Zheng et $\mathrm{al}^{79}$ & 133 & 121 & 26 & $|4|$ & 89 & II & 0.5175 & 72 & 28 & 77 & 23 \\
\hline Yang et al ${ }^{12}$ & 459 & 343 & 78 & 50 & 37 & 7 & 0.9657 & 70 & 30 & 73 & 27 \\
\hline
\end{tabular}

Note: $P_{\text {HWE }}$ represents the $P$-value of Hardy-Weinberg equilibrium test in the genotype distribution of controls.

\section{Frequency of Ser9Gly in the control population}

Pooled frequencies of Ser9Gly stratified by ethnicity were determined for controls. The pooled frequency of Ser9Gly was highest among Latinos (56.8\%; 95\% CI, 55.9-57.6), followed by African-Americans (56.1\%; 95\% CI, 55.3-57.0), East Asians (38.2\%; 95\% CI, 35.0-41.4), Caucasians (29.0\%; 95\% CI, 27.7-30.4), and Indians (22.0\%; 95\% CI, 21.7-22.3).

\section{Quantitative synthesis and heterogeneity analysis}

Pooled ORs and corresponding 95\% CIs were determined for Ser9Gly in the following genetic models: homozygous codominant, heterozygous codominant, dominant, recessive, and allele contrast (Table 3 and Figure 1). The dominant model was found to be most appropriate, according to the principles of genetic model selection. ${ }^{29,86}$ Summary results indicated no association between Ser9Gly and schizophrenia risk. In the dominant model, the pooled OR using a random effects model was 0.950 ( $95 \% \mathrm{CI}, 0.847-1.064 ; P=0.374$ ). Results of subgroup analysis by ethnicity indicated that the Ser9Gly SNP was not associated with schizophrenia among East Asians, Caucasians, or populations evaluated less frequently in the meta-analysis - such as Latino, Indian, and African-American patients (Table 4). Moreover, no association between Ser9Gly and schizophrenia was observed in subgroup analysis according to the source of controls.

\section{Sensitivity analysis}

Sensitivity analysis was carried out to ascertain the contribution of each study to the overall result. Corresponding pooled ORs for analyses in which each of the 73 studies was individually removed indicated that no single study produced a

Table 3 Summarized ORs with 95\% Cls for the association of DRD3 Ser9Gly polymorphism with schizophrenia

\begin{tabular}{|c|c|c|c|c|c|c|c|c|c|}
\hline Polymorphism & Genetic model & $\mathbf{n}$ & Statistical model & OR & $95 \% \mathrm{Cl}$ & $P_{\mathrm{z}}$ & $I^{2}(\%)$ & $\boldsymbol{P}_{\mathrm{h}}$ & $P_{\mathrm{e}}$ \\
\hline \multirow[t]{5}{*}{ Ser9Gly } & Allele contrast & 73 & Random & 0.995 & $0.925-1.069$ & 0.883 & 28.6 & 0.014 & 0.825 \\
\hline & Homozygous codominant & 73 & Random & 0.914 & $0.759-1.102$ & 0.346 & 62.3 & $<0.0001$ & 0.113 \\
\hline & Heterozygous codominant & 73 & Random & 0.838 & $0.716-0.981$ & 0.028 & 47.1 & $<0.0001$ & 0.421 \\
\hline & Dominant & 73 & Random & 0.950 & $0.847-1.064$ & 0.374 & 68.5 & $<0.000 \mathrm{I}$ & 0.040 \\
\hline & Recessive & 73 & Random & 1.139 & $0.965-1.345$ & 0.125 & 57.0 & $<0.000 \mathrm{I}$ & 0.183 \\
\hline
\end{tabular}

Notes: $\mathrm{n}$, number of studies; $P_{\mathrm{z}}, P$-value for association test; $P_{\mathrm{h}}, P$-value for heterogeneity test; $P, P$-value for publication bias test.

Abbreviations: $\mathrm{OR}$, odds ratio; $\mathrm{Cl}$, confidence interval. 


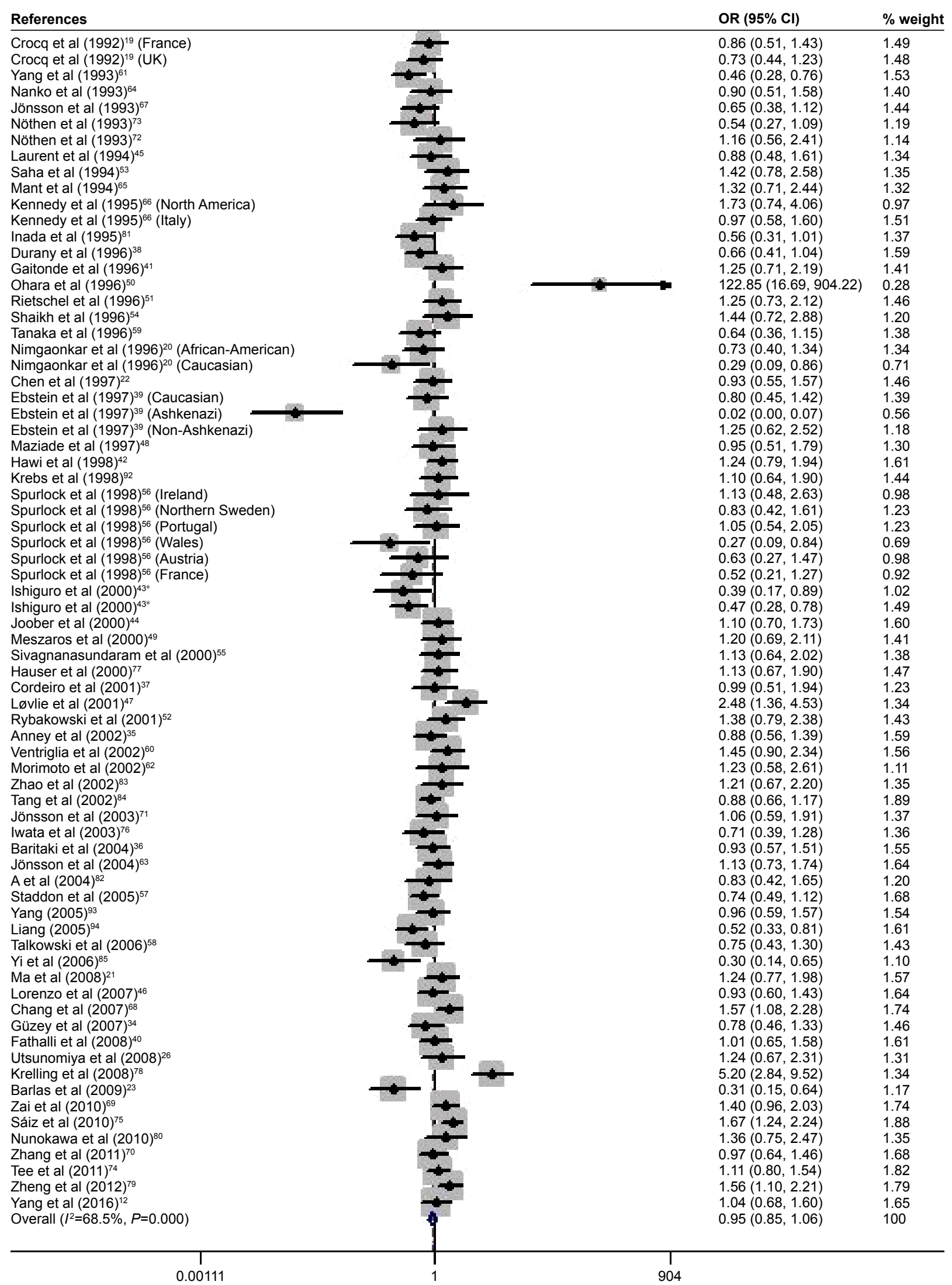

Figure I Forest plot of the association between the Ser9Gly polymorphism of DRD3 and schizophrenia in the dominant genetic model (Ser/Gly + Gly/Gly vs Ser/Ser). Notes: Weights are from random effects analysis. *After the first case-control study, there was a marginally significant association between the Ser 9 Gly polymorphisms and schizophrenia $(P=0.02)$. Thus, these positive findings were replicated in an additional 99 Japanese schizophrenia patients and 132 controls. ${ }^{43}$

Abbreviations: $\mathrm{OR}$, odds ratio; $\mathrm{Cl}$, confidence interval. 
Table 4 Stratified analysis of the association of DRD3 polymorphisms with schizophrenia under dominant model

\begin{tabular}{|c|c|c|c|c|c|c|}
\hline \multirow{2}{*}{$\begin{array}{l}\text { Subgroup } \\
\text { analysis }\end{array}$} & \multicolumn{6}{|c|}{ Ser9Gly } \\
\hline & $\mathbf{n}$ & OR & $95 \% \mathrm{Cl}$ & $P_{\mathrm{z}}$ & $I^{2}(\%)$ & $\boldsymbol{P}_{\mathrm{h}}$ \\
\hline Overall & 73 & 0.950 & $0.847-1.064$ & 0.374 & 68.5 & $<0.0001$ \\
\hline \multicolumn{7}{|l|}{ Ethnicity } \\
\hline East Asians & 25 & 0.915 & $0.75 \mathrm{I}-1.114$ & 0.377 & 72.8 & $<0.0001$ \\
\hline Caucasians & 41 & 0.981 & $0.880-1.094$ & 0.733 & 36.2 & 0.012 \\
\hline Others & 7 & 0.862 & $0.368-2.017$ & 0.732 & 92.2 & $<0.0001$ \\
\hline \multicolumn{7}{|l|}{ Source of controls } \\
\hline Hospital-based & 11 & 1.022 & $0.86|-| .214$ & 0.803 & 4.6 & 0.399 \\
\hline Population-based & 62 & 0.938 & $0.847-1.064$ & 0.334 & 72.0 & $<0.0001$ \\
\hline
\end{tabular}

Notes: $n$, number of studies; $P_{z}, P$-value for association test; $P, P$-value for heterogeneity test. Others included the ethnicities with the rare studies, such as Latino, Indian, and African-American.

Abbreviations: $\mathrm{OR}$, odds ratio; $\mathrm{Cl}$, confidence interval.

significant change in the overall results of the meta-analysis. Hence, these results are stable and reliable.

\section{Publication bias}

A funnel plot was generated to assess potential publication bias (Figure 2), and a small but significant effect of publication bias was detected $\left(P_{\mathrm{e}}=0.040\right)$ (Table 3).

\section{Discussion}

We conducted a meta-analysis of 73 studies (10,634 cases and 11,258 controls) to investigate the potential association of the Ser9Gly SNP in DRD3 with the occurrence of schizophrenia. Our overall findings suggest that no association exists, and results of subgroup analysis stratified by ethnicity and source of controls further validated the distribution disequilibrium of cases and controls.

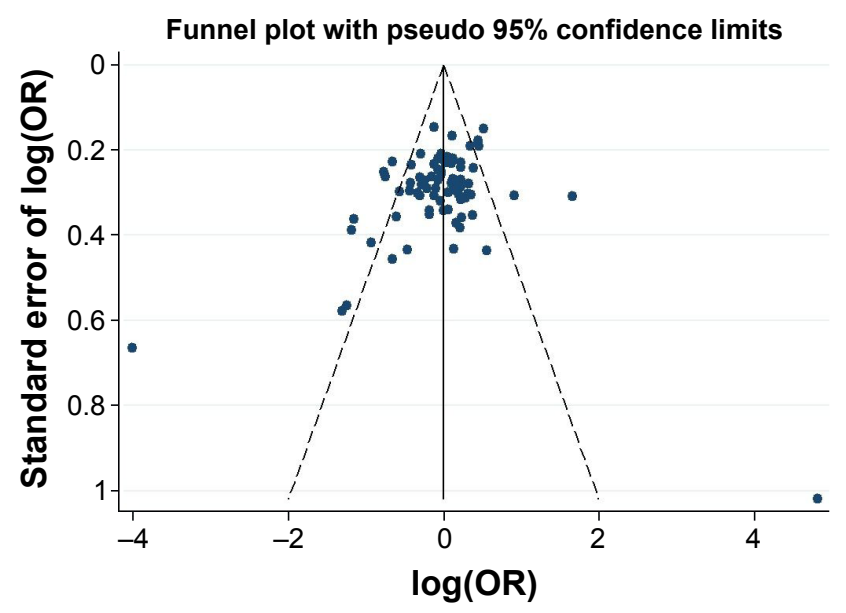

Figure 2 Funnel plot analysis depicting publication bias in the association between the Ser9Gly polymorphism of DRD3 and schizophrenia.

Abbreviation: OR, odds ratio.
Several previous meta-analyses have addressed the putative association between $D R D 3$ polymorphisms and schizophrenia. ${ }^{21,26,71,80,87}$ In general, the results of the current meta-analysis were consistent with those published previously, with the exception of 1 meta-analysis in which $D R D 3$ polymorphisms were found to exert a small but significant effect on schizophrenia susceptibility in Caucasian patients. ${ }^{87}$ Rather than being superfluous, our meta-analysis has several advantages over previous studies. Most importantly, our analysis involved relevant studies that have been published in the interim since the previous meta-analyses were carried out. We included 73 studies that we believe collectively represent $D R D 3$ polymorphisms more accurately than did previous meta-analyses. In addition, we performed subgroup analyses stratified by ethnicity and source of controls to assess potential sources of heterogeneity and to test study stability. Therefore, the results of our study provide a more precise, comprehensive assertion that no association exists between Ser9Gly and schizophrenia.

Some authors have described specific ethnic groups for which associations exist between polymorphisms at certain DRD3 loci and schizophrenia. However, findings of an association of a DRD3 SNP with schizophrenia in 1 population may not be supported in another population. This phenomenon may result from 2 factors. First, different genetic backgrounds may contribute to divergence. The distribution of $D R D 3$ allele frequencies varies among Latinos, African-Americans, East Asians, Caucasians, and Indians. Evidently, genetic liability is a high risk factor for schizophrenia. ${ }^{88} \mathrm{Gly} 9$ allele frequencies vary almost as much in the Japanese control populations $(22 \%-34 \%)$ as they do in northern and western Caucasian control populations $(30 \%-44 \%) .^{71}$ Second, patients from different populations may have disparate lifestyles and may be affected by different environmental factors. ${ }^{89}$ Epigenetic modifications that contribute to schizophrenia may be a product of transregulatory or environmental risk factors. ${ }^{90}$

The relatively small sample sizes of Latino, AfricanAmerican, Indian, Ashkenazi, and non-Ashkenazi patients limited our ability to isolate stable effects for these subgroups. More studies need to be performed to explore the association between Ser9Gly polymorphism and the risk of schizophrenia in these above populations. Moreover, the lack of an association between Ser9Gly and schizophrenia was upheld when the analysis was stratified by the source of controls. However, control patients in hospital-based studies do not necessarily represent the general population, particularly when the polymorphism being evaluated is related 
to a disorder that affects hospital-based control patients. ${ }^{91}$ Thus, the negative results by the source of controls should be interpreted carefully. Because this Gly allele is known to alter dopamine-binding affinity, it can, to some degree, influence the function of dopamine neurotransmitter. Thus, more effort is needed to explore whether it is involved in the risk of schizophrenia.

The present study had several limitations. We observed significant heterogeneity in overall and subgroup analyses. Although we performed subgroup analysis to investigate potential sources of heterogeneity, no single factor completely accounted for this heterogeneity. Therefore, other unidentified aspects might partially contribute to heterogeneity. Second, we detected a slight but significant publication bias in the included studies. This bias can be explained, in part, by our inclusion of only English- and Chinese-language studies. Another main reason is that the negative results are not easier to publish than the positive results. Third, genegene interactions and epigenetics were not examined in this meta-analysis, owing to insufficient information in the included studies. By evaluating only $1 \mathrm{SNP}$ in $D R D 3$, we may have limited our analysis to a polymorphism that plays a minute role in the overall genetic influences of schizophrenia. This disorder is thought to arise from the mutual influence of multiple genes.

In summary, we found no evidence of an association between the Ser9Gly SNP in DRD3 and risk of schizophrenia. Studies involving larger sample sizes will be necessary to confirm the results of this meta-analysis - especially for certain ethnic subpopulations - and to address the epigenetic mechanisms and environmental influences that contribute to schizophrenia risk.

\section{Acknowledgments}

This study was supported by grants from the National Natural Science Foundation of China (81601653) and the Doctoral Research Start Foundation of Liaoning Province (201601115) for Dr Jun Yao.

\section{Author contributions}

All authors contributed toward data analysis, drafting and critically revising the paper, gave final approval of the version to be published, and agree to be accountable for all aspects of the work.

\section{Disclosure}

The authors report no conflicts of interest in this work.

\section{References}

1. Hoenders R, Bartels-Velthuis A, Vollbehr N, Bruggeman R, Knechtering R, de Jong J. Natural medicines in schizophrenia: a systematic review. J Altern Complement Med. 2014;20(5):A79.

2. Mueser KT, McGurk SR. Schizophrenia. Lancet. 2004;363(9426): 2063-2072.

3. Heron EA, Cormican P, Donohoe G, et al. No evidence that runs of homozygosity are associated with schizophrenia in an Irish genomewide association dataset. Schizophr Res. 2014;154(1-3):79-82.

4. Sullivan PF, Kendler KS, Neale MC. Schizophrenia as a complex trait: evidence from a meta-analysis of twin studies. Arch Gen Psychiatry. 2003;60(12):1187-1192.

5. Wan CL, Zainal NZ, Lian LH, Mohamed Z. Association of the functional polymorphism in the catechol-O-methyltransferase gene with schizophrenia in the three ethnic groups of the Malaysian population. Psychiatry Res. 2011;189(1):67-71.

6. Yuan J, Jin C, Sha W, et al. A competitive PCR assay confirms the association of a copy number variation in the VIPR2 gene with schizophrenia in Han Chinese. Schizophr Res. 2014;156(1):66-70.

7. Abi-Dargham A, Moore H. Prefrontal DA transmission at D1 receptors and the pathology of schizophrenia. Neuroscientist. 2003;9(5): 404-416.

8. Fan H, Zhang F, Xu Y, et al. An association study of DRD2 gene polymorphisms with schizophrenia in a Chinese Han population. Neurosci Lett. 2010;477(2):53-56.

9. Howes OD, Kapur S. The dopamine hypothesis of schizophrenia: version III - the final common pathway. Schizophr Bull. 2009;35(3): 549-562.

10. Davis J, Moylan S, Harvey BH, Maes M, Berk M. Neuroprogression in schizophrenia: pathways underpinning clinical staging and therapeutic corollaries. Aust N Z J Psychiatry. 2014;48(6):512-529.

11. Sokoloff P, Giros B, Martres MP, et al. Localization and function of the D3 dopamine receptor. Arzneimittelforschung. 1992;42(2A):224-230.

12. Yang B, Niu W, Chen S, et al. Association study of dopamine receptor genes polymorphisms with the risk of schizophrenia in the Han Chinese population. Psychiatry Res. 2016;245:361-364.

13. Utsunomiya K, Shinkai T, Sakata S, et al. Genetic association between the dopamine D3 receptor gene polymorphism (Ser9Gly) and tardive dyskinesia in patients with schizophrenia: a reevaluation in East Asian populations. Neurosci Lett. 2012;507(1):52-56.

14. Savitz J, Hodgkinson CA, Martin-Soelch C, et al. The functional DRD3 Ser9Gly polymorphism (rs6280) is pleiotropic, affecting reward as well as movement. PLoS One. 2013;8(1):e54108.

15. Jhun E, He Y, Yao Y, Molokie RE, Wilkie DJ, Wang ZJ. Dopamine D3 receptor Ser9Gly and catechol-o-methyltransferase Val158Met polymorphisms and acute pain in sickle cell disease. Anesth Analg. 2014;119(5):1201-1207.

16. Chang TT, Chen SL, Chang YH, et al. The DRD3 Ser9Gly polymorphism predicted metabolic change in drug-naive patients with bipolar II disorder. Medicine (Baltimore). 2016;95(24):e3488.

17. Xu S, Liu J, Yang X, Qian Y, Xiao Q. Association of the DRD2 CAnSTR and DRD3 Ser9Gly polymorphisms with Parkinson's disease and response to dopamine agonists. J Neurol Sci. 2017;372:433-438.

18. Zai CC, Manchia M, Sonderby IE, et al. Investigation of the genetic interaction between BDNF and DRD3 genes in suicidical behaviour in psychiatric disorders. World J Biol Psychiatry. 2015;16(3): 171-179.

19. Crocq MA, Mant R, Asherson P, et al. Association between schizophrenia and homozygosity at the dopamine D3 receptor gene. $J$ Med Genet. 1992;29(12):858-860.

20. Nimgaonkar VL, Sanders AR, Ganguli R, et al. Association study of schizophrenia and the dopamine D3 receptor gene locus in two independent samples. Am J Med Genet. 1996;67(6):505-514.

21. Ma G, He Z, Fang W, et al. The Ser9Gly polymorphism of the dopamine D3 receptor gene and risk of schizophrenia: an association study and a large meta-analysis. Schizophr Res. 2008;101(1-3):26-35. 
22. Chen CH, Liu MY, Wei FC, Koong FJ, Hwu HG, Hsiao KJ. Further evidence of no association between Ser9Gly polymorphism of dopamine D3 receptor gene and schizophrenia. Am J Med Genet. 1997;74(1):40-43.

23. Barlas IO, Cetin M, Erdal ME, et al. Lack of association between DRD3 gene polymorphism and response to clozapine in Turkish schizoprenia patients. Am J Med Genet B Neuropsychiatr Genet. 2009;150B(1):56-60.

24. Yao J, Pan YQ, Ding M, Pang H, Wang BJ. Association between DRD2 (rs1799732 and rs1801028) and ANKK1 (rs1800497) polymorphisms and schizophrenia: a meta-analysis. Am J Med Genet B Neuropsychiatr Genet. 2015;168B(1):1-13.

25. Yang B, Fan S, Zhi X, et al. Associations of MTHFR gene polymorphisms with hypertension and hypertension in pregnancy: a metaanalysis from 114 studies with 15411 cases and 21970 controls. PLoS One. 2014;9(2):e87497.

26. Utsunomiya K, Shinkai T, De Luca V, et al. Genetic association between the dopamine D3 gene polymorphism (Ser9Gly) and schizophrenia in Japanese populations: evidence from a case-control study and metaanalysis. Neurosci Lett. 2008;444(2):161-165.

27. Thakkinstian A, McEvoy M, Minelli C, et al. Systematic review and meta-analysis of the association between \{beta\}2-adrenoceptor polymorphisms and asthma: a HuGE review. Am J Epidemiol. 2005; 162(3):201-211.

28. Munafò MR, Flint J. Meta-analysis of genetic association studies. Trends Genet. 2004;20(9):439-444.

29. Thakkinstian A, McElduff P, D'Este C, Duffy D, Attia J. A method for meta-analysis of molecular association studies. Stat Med. 2005; 24(9):1291-1306

30. Higgins JP, Thompson SG, Deeks JJ, Altman DG. Measuring inconsistency in meta-analyses. BMJ. 2003;327(7414):557-560.

31. Zintzaras E, Ioannidis JP. Heterogeneity testing in meta-analysis of genome searches. Genet Epidemiol. 2005;28(2):123-137.

32. Higgins JP, Thompson SG. Quantifying heterogeneity in a metaanalysis. Stat Med. 2002;21(11):1539-1558.

33. Egger M, Davey Smith G, Schneider M, Minder C. Bias in metaanalysis detected by a simple, graphical test. $B M J .1997 ; 315(7109)$ : 629-634.

34. Güzey C, Scordo MG, Spina E, Landsem VM, Spigset O. Antipsychoticinduced extrapyramidal symptoms in patients with schizophrenia: associations with dopamine and serotonin receptor and transporter polymorphisms. Eur J Clin Pharmacol. 2007;63(3):233-241.

35. Anney RJ, Rees MI, Bryan E, et al. Characterisation, mutation detection, and association analysis of alternative promoters and $5^{\prime}$ UTRs of the human dopamine D3 receptor gene in schizophrenia. Mol Psychiatry. 2002;7(5):493-502.

36. Baritaki S, Rizos E, Zafiropoulos A, et al. Association between schizophrenia and DRD3 or HTR2 receptor gene variants. Eur J Hum Genet. 2004;12(7):535-541.

37. Cordeiro Q Jr, Junqueira R, Vallada H. Estudo de associação entre o polimorfismo serina-9-glicina do receptor dopaminérgico D3 e esquizofrenia [Study of association between the ser-9-gly polymorphism of the D3 dopaminergic receptor and schizophrenia]. Arq Neuropsiquiatr. 2001;59(2-A):219-222. Portuguese [with English abstract].

38. Durany N, Thome J, Palomo A, Foley P, Riederer P, Cruz-Sanchez FF. Homozygosity at the dopamine D3 receptor gene in schizophrenic patients. Neurosci Lett. 1996;220(3):151-154.

39. Ebstein RP, Macciardi F, Heresco-Levi U, et al. Evidence for an association between the dopamine D3 receptor gene DRD3 and schizophrenia. Hum Hered. 1997;47(1):6-16.

40. Fathalli F, Rouleau GA, Xiong L, et al. No association between the DRD3 Ser9Gly polymorphism and schizophrenia. Schizophr Res. 2008;98(1-3):98-104.

41. Gaitonde EJ, Morris A, Sivagnanasundaram S, McKenna PJ, Hunt DM, Mollon JD. Assessment of association of D3 dopamine receptor MscI polymorphism with schizophrenia: analysis of symptom ratings, family history, age at onset, and movement disorders. Am J Med Genet. 1996; 67(5):455-458.
42. Hawi Z, McCabe U, Straub RE, et al. Examination of new and reported data of the DRD3/MscI polymorphism: no support for the proposed association with schizophrenia. Mol Psychiatry. 1998;3(2):150-155.

43. Ishiguro H, Okuyama Y, Toru M, Arinami T. Mutation and association analysis of the $5^{\prime}$ region of the dopamine D3 receptor gene in schizophrenia patients: identification of the Ala38Thr polymorphism and suggested association between DRD3 haplotypes and schizophrenia. Mol Psychiatry. 2000;5(4):433-438.

44. Joober R, Toulouse A, Benkelfat C, et al. DRD3 and DAT1 genes in schizophrenia: an association study. $J$ Psychiatr Res. 2000;34(4-5): 285-291.

45. Laurent C, Savoye C, Samolyk D, et al. Homozygosity at the dopamine D3 receptor locus is not associated with schizophrenia. J Med Genet. 1994;31(3):260.

46. Lorenzo CV, Baca-Garcia E, Hernandez MD, et al. No association between the Ser9Gly polymorphism of the dopamine D3 receptor gene and schizophrenia in a Spanish sample. Am J Med Genet B Neuropsychiatr Genet. 2007;144B(3):344-346.

47. Løvlie R, Thara R, Padmavathi R, Steen VM, McCreadie RG. Ser9Gly dopamine D3 receptor polymorphism and spontaneous dyskinesia in never-medicated schizophrenic patients. Mol Psychiatry. 2001;6(1):6-7.

48. Maziade M, Martinez M, Rodrigue C, et al. Childhood/early adolescence-onset and adult-onset schizophrenia. Heterogeneity at the dopamine D3 receptor gene. Br J Psychiatry. 1997;170:27-30.

49. Meszaros K, Lenzinger E, Hornik K, et al. Association study of schizophrenia spectrum disorders and dopamine D3 receptor gene: is schizoaffective disorder special? Psychiatry Res. 2000;96(2):179-183.

50. Ohara K, Nakamura Y, Xie DW, et al. Polymorphisms of dopamine D2-like (D2, D3, and D4) receptors in schizophrenia. Biol Psychiatry. 1996;40(12):1209-1217.

51. Rietschel M, Nothen MM, Albus M, et al. Dopamine D3 receptor Gly9/Ser9 polymorphism and schizophrenia: no increased frequency of homozygosity in German familial cases. Schizophr Res. 1996;20(1-2): 181-186.

52. Rybakowski JK, Borkowska A, Czerski PM, Hauser J. Dopamine D3 receptor (DRD3) gene polymorphism is associated with the intensity of eye movement disturbances in schizophrenic patients and healthy subjects. Mol Psychiatry. 2001;6(6):718-724.

53. Saha N, Tsoi WF, Low PS, Basair J, Tay JS. Lack of association of the dopamine D3 receptor gene polymorphism (Ball) in Chinese schizophrenic males. Psychiatr Genet. 1994;4(4):201-204.

54. Shaikh S, Collier DA, Sham PC, et al. Allelic association between a Ser-9-Gly polymorphism in the dopamine D3 receptor gene and schizophrenia. Hum Genet. 1996;97(6):714-719.

55. Sivagnanasundaram S, Morris AG, Gaitonde EJ, McKenna PJ, Mollon JD, Hunt DM. A cluster of single nucleotide polymorphisms in the 5'-leader of the human dopamine D3 receptor gene (DRD3) and its relationship to schizophrenia. Neurosci Lett. 2000;279(1):13-16.

56. Spurlock G, Williams J, McGuffin P, et al. European multicentre association study of schizophrenia: a study of the DRD2 Ser311Cys and DRD3 Ser9Gly polymorphisms. Am J Med Genet. 1998;81(1):24-28.

57. Staddon S, Arranz MJ, Mancama D, et al. Association between dopamine D3 receptor gene polymorphisms and schizophrenia in an isolate population. Schizophr Res. 2005;73(1):49-54.

58. Talkowski ME, Mansour H, Chowdari KV, et al. Novel, replicated associations between dopamine $\mathrm{D} 3$ receptor gene polymorphisms and schizophrenia in two independent samples. Biol Psychiatry. 2006;60(6):570-577.

59. Tanaka T, Igarashi $\mathrm{S}$, Onodera $\mathrm{O}$, et al. Association study between schizophrenia and dopamine D3 receptor gene polymorphism. Am J Med Genet. 1996;67(4):366-368.

60. Ventriglia M, Bocchio Chiavetto L, Bonvicini C, et al. Allelic variation in the human prodynorphin gene promoter and schizophrenia. Neuropsychobiology. 2002;46(1):17-21.

61. Yang L, Li T, Wiese C, et al. No association between schizophrenia and homozygosity at the $\mathrm{D} 3$ dopamine receptor gene. Am J Med Genet. 1993;48(2):83-86. 
62. Morimoto K, Miyatake R, Nakamura M, Watanabe T, Hirao T, Suwaki H. Delusional disorder: molecular genetic evidence for dopamine psychosis. Neuropsychopharmacology. 2002;26(6):794-801.

63. Jönsson EG, Kaiser R, Brockmöller J, Nimgaonkar VL, Crocq MA. Meta-analysis of the dopamine D3 receptor gene (DRD3) Ser9Gly variant and schizophrenia. Psychiatr Genet. 2004;14(1):9-12.

64. Nanko S, Sasaki T, Fukuda R, et al. A study of the association between schizophrenia and the dopamine D3 receptor gene. Hum Genet. 1993; 92(4):336-338.

65. Mant R, Williams J, Asherson P, Parfitt E, McGuffin P, Owen MJ. Relationship between homozygosity at the dopamine $\mathrm{D} 3$ receptor gene and schizophrenia. Am J Med Genet. 1994;54(1):21-26.

66. Kennedy JL, Billett EA, Macciardi FM, et al. Association study of dopamine D3 receptor gene and schizophrenia. Am J Med Genet. 1995; 60(6):558-562.

67. Jönsson E, Lannfelt L, Sokoloff P, Schwartz JC, Sedvall G. Lack of association between schizophrenia and alleles in the dopamine D3 receptor gene. Acta Psychiatr Scand. 1993;87(5):345-349.

68. Chang HA, Lu RB, Lin WW, et al. Lack of association between dopamine D3 receptor Ser9Gly polymorphism and schizophrenia in Han Chinese population. Acta Neuropsychiatr. 2007;19(6): 344-350.

69. Zai CC, Manchia M, De Luca V, et al. Association study of BDNF and DRD3 genes in schizophrenia diagnosis using matched case-control and family based study designs. Prog Neuropsychopharmacol Biol Psychiatry. 2010;34(8):1412-1418.

70. Zhang F, Fan H, Xu Y, et al. Converging evidence implicates the dopamine D3 receptor gene in vulnerability to schizophrenia. Am J Med Genet B Neuropsychiatr Genet. 2011;156B(5):613-619.

71. Jönsson EG, Flyckt L, Burgert E, et al. Dopamine D3 receptor gene Ser9Gly variant and schizophrenia: association study and meta-analysis. Psychiatr Genet. 2003;13(1):1-12.

72. Nöthen MM, Cichon S, Propping P, Fimmers R, Schwab SG, Wildenauer DB. Excess of homozygosity at the dopamine D3 receptor gene in schizophrenia not confirmed. J Med Genet. 1993;30(8):708.

73. Nöthen MM, Körner J, Lannfelt L, et al. Lack of association between schizophrenia and alleles of the dopamine D1, D2, D3 and D4 receptor loci. Psychiatr Genet. 1993;3:89-94.

74. Tee S, Tang P, Loh H. Genetic association analysis of dopamine DRD3 Ser9Gly polymorphism and schizophrenia in Malay population. Iran J Public Health. 2011;40(2):6-10.

75. Sáiz PA, Garcia-Portilla MP, Arango C, et al. Genetic polymorphisms in the dopamine-2 receptor (DRD2), dopamine-3 receptor (DRD3), and dopamine transporter (SLC6A3) genes in schizophrenia: data from an association study. Prog Neuropsychopharmacol Biol Psychiatry. 2010;34(1):26-31.

76. Iwata Y, Matsumoto H, Minabe Y, et al. Early-onset schizophrenia and dopamine-related gene polymorphism. Am J Med Genet B Neuropsychiatr Genet. 2003;116B(1):23-26.

77. Hauser J, Czerski PM, Czarny-Ratajczak M, et al. Brak asocjacji pomiędzy polimorfizmem genów DRD2 i DRD3 a schizofrenią [Lack of association between polymorphisms of DRD2 and DRD3 genes and schizophrenia]. Post Psychiatr Neurol. 2000;9(2):149-157. Polish [with English abstract].
78. Krelling R, Cordeiro Q, Miracca E, et al. Molecular genetic casecontrol women investigation from the first Brazilian high-risk study on functional psychosis. Rev Bras Psiquiatr. 2008;30(4):341-345.

79. Zheng C, Shen Y, Xu Q. Rs1076560, a functional variant of the dopamine D2 receptor gene, confers risk of schizophrenia in Han Chinese. Neurosci Lett. 2012;518(1):41-44.

80. Nunokawa A, Watanabe Y, Kaneko N, et al. The dopamine D3 receptor (DRD3) gene and risk of schizophrenia: case-control studies and an updated meta-analysis. Schizophr Res. 2010;116(1):61-67.

81. Inada T, Sugita T, Dobashi I, et al. Dopamine D3 receptor gene polymorphism and the psychiatric symptoms seen in first-break schizophrenic patients. Psychiatr Genet. 1995;5(3):113-116.

82. A Z, Li H, Ma ZM, Zhang L. An association study of polymorphism of DRD3 gene with schizophrenia in Chinese Han population. J Dali Coll. 2004;3(1):3-4. Chinese.

83. Zhao Z, Shi J, Dai J, et al. An association study between a Ser-9-Gly polymorphism in the dopamine D3 receptor gene and schizophrenia. Health Psychol J. 2002;10(3):163-165. Chinese.

84. Tang YL, Wang YF, Cai ZJ, Zhou RL, Zhou CF. [Association study of dopamine D3 receptor gene with schizophrenia subtypes]. Chin J Psychiatry. 2002;35(1):11-14. Chinese [with English abstract].

85. Yi Y, Wang JK, Wang MJ, Lin D, Qing HP. Association between Ser9Gly polymorphism in dopamine D3 receptor gene and schizophrenia. J Clin Psychol Med. 2006;16(2):73-75. Chinese.

86. Arj-Ong S, Thakkinstian A, McEvoy M, Attia J. A systematic review and meta-analysis of tumor necrosis factor $\alpha-308$ polymorphism and Kawasaki disease. Pediatr Int. 2010;52(4):527-532.

87. Dubertret C, Gorwood P, Ades J, Feingold J, Schwartz JC, Sokoloff P. Meta-analysis of DRD3 gene and schizophrenia: ethnic heterogeneity and significant association in Caucasians. Am J Med Genet. 1998;81(4): 318-322.

88. Walder DJ, Faraone SV, Glatt SJ, Tsuang MT, Seidman LJ. Genetic liability, prenatal health, stress and family environment: risk factors in the Harvard Adolescent Family High Risk for Schizophrenia Study. Schizophr Res. 2014;157(1-3):142-148.

89. Frey S. The economic burden of schizophrenia in Germany: a population-based retrospective cohort study using genetic matching. Eur Psychiatry. 2014;29(8):479-489.

90. Walton E, Liu J, Hass J, et al. MB-COMT promoter DNA methylation is associated with working-memory processing in schizophrenia patients and healthy controls. Epigenetics. 2014;9(8):1101-1107.

91. Ruano-Ravina A, Pérez-Ríos M, Barros-Dios JM. Population-based versus hospital-based controls: are they comparable? Gac Sanit. 2008; 22(6):609-613.

92. Krebs MO, Sautel F, Bourdel MC, et al. Dopamine D3 receptor gene variants and substance abuse in schizophrenia. Mol Psychiatry. 1998;3(4):337-341.

93. Yang H. [The association between DRD3 Ser9Gly polymorphism and schizophrenia] [master's thesis]. Shenyang: China Medical University; 2005. Chinese.

94. Liang KW. [The association of DA2, DA3, and DA4 receptor gene polymorphology with schizophrenia also in medical jurisprudence and human genetics studies] [doctor's thesis]. Shenyang: China Medical University; 2005. Chinese.
Neuropsychiatric Disease and Treatment

\section{Publish your work in this journal}

Neuropsychiatric Disease and Treatment is an international, peerreviewed journal of clinical therapeutics and pharmacology focusing on concise rapid reporting of clinical or pre-clinical studies on a range of neuropsychiatric and neurological disorders. This journa is indexed on PubMed Central, the 'PsycINFO' database and CAS,

\section{Dovepress}

and is the official journal of The International Neuropsychiatric Association (INA). The manuscript management system is completely online and includes a very quick and fair peer-review system, which is all easy to use. Visit http://www.dovepress.com/testimonials.php to read real quotes from published authors. 